\title{
Morphology control of molecular assemblies prepared from bio-based amphiphilic polymers with a helical hydrophobic unit and application as nanocarriers for contrast agents and/or drug delivery
}

\begin{abstract}
Akira Makino $^{1,2}$
Bio-based amphiphilic polymers with a helical hydrophobic unit have been studied extensively with regard to molecular assemblies and their morphology. To use specific characteristics of the helical units, such as coiled-coil and stereocomplex formation between the right- and left-handed helices, morphologies more complex than conventional tubes and vesicles must be prepared. Membranes based on stereocomplex formation were sufficiently stable to induce phase separation, which led to a unique self-assembly, 'patchwork self-assembly'. The molecular assemblies generated were thermodynamically stable and, in certain cases, exhibited membrane fluidity because the regularly aligned helical hydrophobic units contribute to the molecular assembly and allow for translational diffusion of the helices in the assemblies. The molecular assembly physical properties can be finely tuned, and the assemblies were used as carriers for drug delivery systems (DDSs). The lactosome is a polymeric micelle $35 \mathrm{~nm}$ in diameter. In in vivo experiments using transplanted tumor model mice, the lactosome selectively accumulated at tumor regions through an enhanced permeability and retention (EPR) effect, and its entrapment by self-defense systems of living organisms was significantly suppressed. Therefore, the lactosome is likely an excellent carrier for DDS.
\end{abstract}

Polymer Journal (2014) 46, 783-791; doi:10.1038/pj.2014.73; published online 13 August 2014

\section{INTRODUCTION}

Drug delivery systems (DDSs) compose an important technology that improves the efficiency of encapsulated drug delivery to targeted regions and suppresses side effects. Various types of nanoparticles, including organic and inorganic materials, were developed as DDS carriers. ${ }^{1-5}$ Among the carriers, the liposome, which is a vesicular assembly of phospholipids, has been actively examined, and certain drugs using liposomes are commercially available. ${ }^{6,7}$ The liposome is an excellent DDS carrier; however, certain weak features must be improved. For example, in vivo liposome stability is relatively low owing to weak intermolecular hydrophobic interactions between hydrophobic alkyl chains. Theoretically, the morphology formed is determined by the packing parameters; ${ }^{8}$ however, assemblies with complex morphologies are rarely prepared. Recently, size, morphology and softness of the molecular assembly have been considered important factors for improving drug delivery and cell uptake efficiency; ${ }^{9,10}$ therefore, to develop new strategies for molecular assembly preparation, such factors are considered essential.

One approach for preparing molecular assemblies with greater stability than liposomes uses a polymersome, which is prepared from an amphiphilic synthetic block polymer. ${ }^{11,12}$ The long hydrophobic chain yields strong intermolecular hydrophobic interactions but introduces polymer entanglement into the assembly. Therefore, the resulting assemblies are thermodynamically stable, but lack membrane fluidity, which is one of the most important characteristics of a cell membrane.

Specific oligo- and polypeptide chains can form higher-ordered structures. Among them, the author focused on hydrophobic helical oligopeptide chains. Owing to intermolecular hydrophobic and dipole-dipole interactions, helical hydrophobic polymers are regularly aligned in the assemblies and form assemblies that include micelles, vesicles and single assembled monolayers. ${ }^{13-15}$ Micelle and vesicle preparation from amphiphilic polypeptides began in the late 1990s, and they are referred to as 'peptosomes. ${ }^{16-18}$ A peptosome is more thermodynamically stable than a liposome and exhibits membrane fluidity because intermolecular polymer entanglement is avoided.

Recently, functionalized molecular assemblies prepared from amphiphilic polypeptides (polydepsipeptide) with a helical hydrophobic component were actively examined, and various stimulusresponsive and morphologically controlled assemblies have been prepared. This paper focuses on recent developments in molecular assembly preparation and utilization in medicinal fields.

${ }^{1}$ Biomedical Imaging Research Center, University of Fukui, Fukui, Japan and ${ }^{2}$ Research and Education Program for Life Science, University of Fukui, Fukui, Japan Correspondence: Dr A Makino, Biomedical Imaging Research, University of Fukui, 23-3 Matsuoka-shimoaizuki, Eiheiji-cho, Yoshida-gun, Fukui 910-1193, Japan. E-mail: amakino@u-fukui.ac.jp

Received 19 May 2014; revised 22 June 2014; accepted 23 June 2014; published online 13 August 2014 


\section{MOLECULAR ASSEMBLIES WITH UNIQUE PROPERTIES FROM AN AMPHIPHILE-CONTAINING HELICAL HYDROPHOBIC BLOCK}

Stimulus-responsive molecular assembly (pH-responsive assembly) design

One of the greatest advantages of polymer-based nanomaterials is that they can be easily functionalized through sequence design. Therefore, researchers have prepared various types of $\mathrm{pH}-$-, photo- and thermosensitive assemblies.

Two basic approaches have been used to design $\mathrm{pH}$-sensitive assemblies. One includes selecting a $\mathrm{pH}$-sensitive residue as a hydrophobic and/or hydrophilic block in the amphiphilic polypeptide. Representative $\mathrm{pH}$-sensitive peptide sequences that form a helical structure include L-glutamic acid (Glu) and L-lysine (Lys) repeats. Rodriguez-Hernandez and Lecommandoux et al. ${ }^{19}$ prepared 'schizophrenic' vesicles from poly(L-glutamic acid)-b-poly(L-lysine) with two oppositely charged polypeptide blocks. In aqueous solution at $\mathrm{pH}=3$ and 12 , the polypeptide formed vesicles with the diameters ca. 110 and $175 \mathrm{~nm}$, respectively, and a narrow size distribution (Figure 1a). Vesicle formation is related to the systematic presence of a polypeptide in a rod-like helical conformation in the hydrophobic portion of the membrane. ${ }^{19}$ Furthermore, the 'schizophrenic' aggregation of linear-dendron-type polyampholyte, poly(L-lysine)- $b$ -
D2-poly(L-glutamic acid), where D2 is the second generation of a poly(amido amine), was examined by varying the solution $\mathrm{pH}$. Large compound micelles and worm-like micelles with a PLGA core were observed at $\mathrm{pH}$ values below the isoelectric point, whereas large compound vesicles, simple vesicles and tubular structures with a PLL core were obtained at $\mathrm{pH}$ values above the isoelectric point. ${ }^{20}$

The other approach is to insert a $\mathrm{pH}$-sensitive unit into the amphiphilic polypeptide (Figure 1b). For example, amphiphilic polypeptides with a histidine residue between helical hydrophobic and hydrophilic poly(sarcosine) (poly(Sar)) units (S26 3 HisL12) were synthesized, and their morphologic changes were examined by changing the $\mathrm{pH}$ conditions of the solution. At a neutral $\mathrm{pH}$, the amphiphile formed a roll sheet assembly. Under acidic $\mathrm{pH}$ conditions below the His residue $\mathrm{pKa}$, regularly aligned helix packing in the assembly was looser owing to electrostatic repulsion, and the roll sheet layer spacing was broadened. ${ }^{21}$ The morphologic changes of the amphiphile with one His residue (S23 ${ }_{3}$ HisL12) were compared with an amphiphile containing two His residues (S26 ${ }_{3}$ His2L12); S26 3 His2L12 showed a sharp transformation with the change in $\mathrm{pH}$, and a tubular structure under neutral conditions was transformed into a helical ribbon ( $\mathrm{pH}$ 5.0) and a twisted ribbon $\left(\mathrm{pH}\right.$ 3.0). ${ }^{22}$ As discussed above, the helical hydrophobic unit does not produce intermolecular polymer entanglements in the assembly.

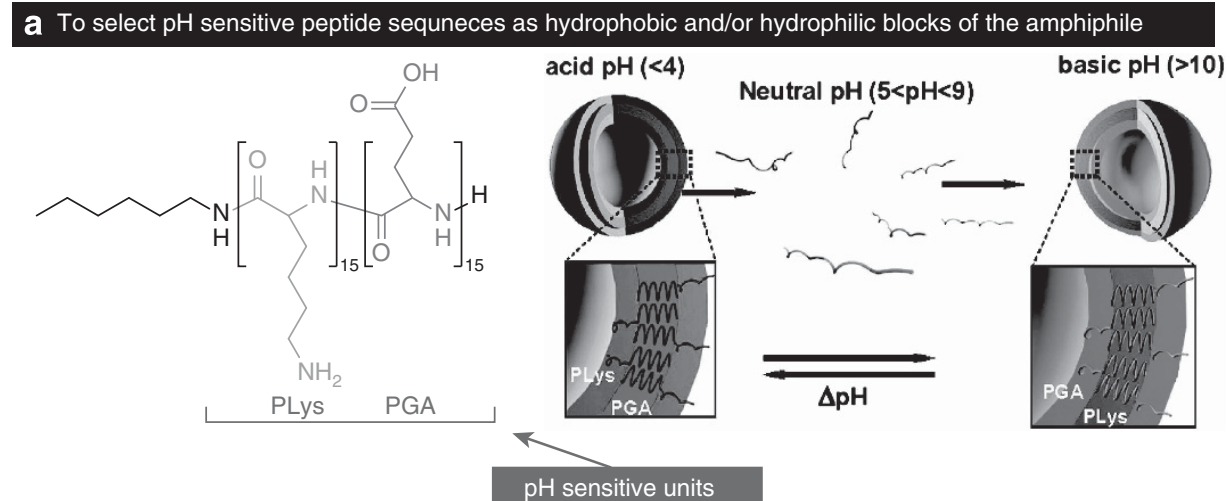

b To insert $\mathrm{pH}$ sensitive unit into the amphiphile<smiles>CCCNC(=O)CNC(=O)CNC(=O)CNC(C)=O</smiles><smiles>COC(=O)C(C)(C)NC(=O)C1CC(C)CCN1</smiles>

S26 ${ }_{3}$ HisL12<smiles>CC(=O)CNC(=O)CO</smiles>

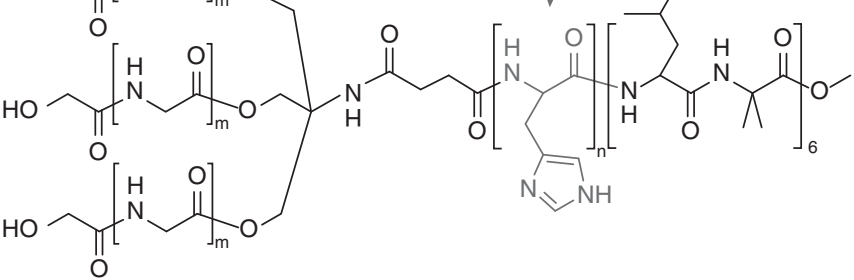

S23 ${ }_{3}$ HisL12: $m=23, n=1$

S26 ${ }_{3}$ His2L12: $m=26, n=2$

Figure 1 Two approaches for preparing pH-sensitive molecular assemblies. (a) Using the $\mathrm{pH}$-sensitive polypeptide sequence as a component of the amphiphile. (b) A pH-sensitive unit such as a His residue is inserted between the hydrophilic and helical hydrophobic components of the amphiphilic polypeptide. (reprinted with permission from Figure 1 in Rodriguez-Hernandez and Lecommandoux ${ }^{19}$ Copyright 2008 American Chemical Society). A full color version of this figure is available at Polymer Journal online. 

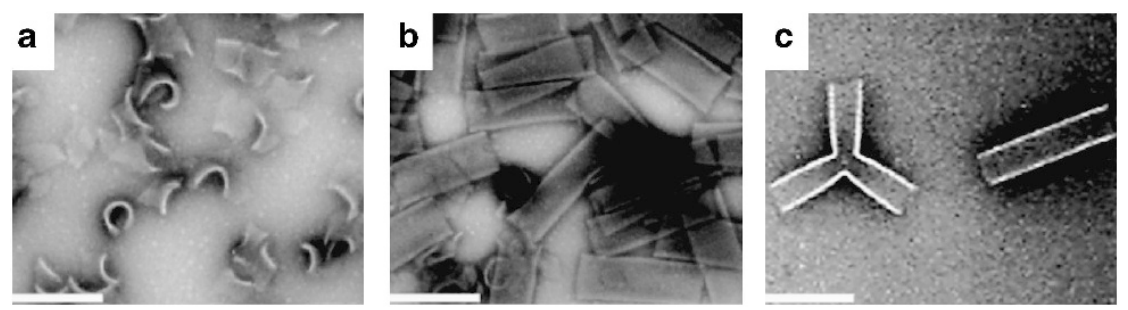

Figure 2 Transmission electron microscopy (TEM) images of (Sar) $27_{-b}-\left(\right.$ Leu-Aib) 6 (a) before and (b) after heating the dispersion at $90^{\circ} \mathrm{C}$ for $10 \mathrm{~min}$ as well as (c) of a $7 / 3\left(\mathrm{~mol} \mathrm{~mol}^{-1}\right)$ mixture of $(\text { Sar })_{27}-b$-(Leu-Aib) 6 and $(\text { Sar })_{10}-b$-(Leu-Aib) 6 after heating the dispersion at $90^{\circ} \mathrm{C}$ for $10 \mathrm{~min}$. The images were negatively stained using 2\% uranyl acetate. Scale bar: $200 \mathrm{~nm}$ (reprinted with permission from Figures 4 and 7 in Kanzaki et al..27 Copyright 2008 WILEY-VCH Verlag GmbH \& Co. KGaA).
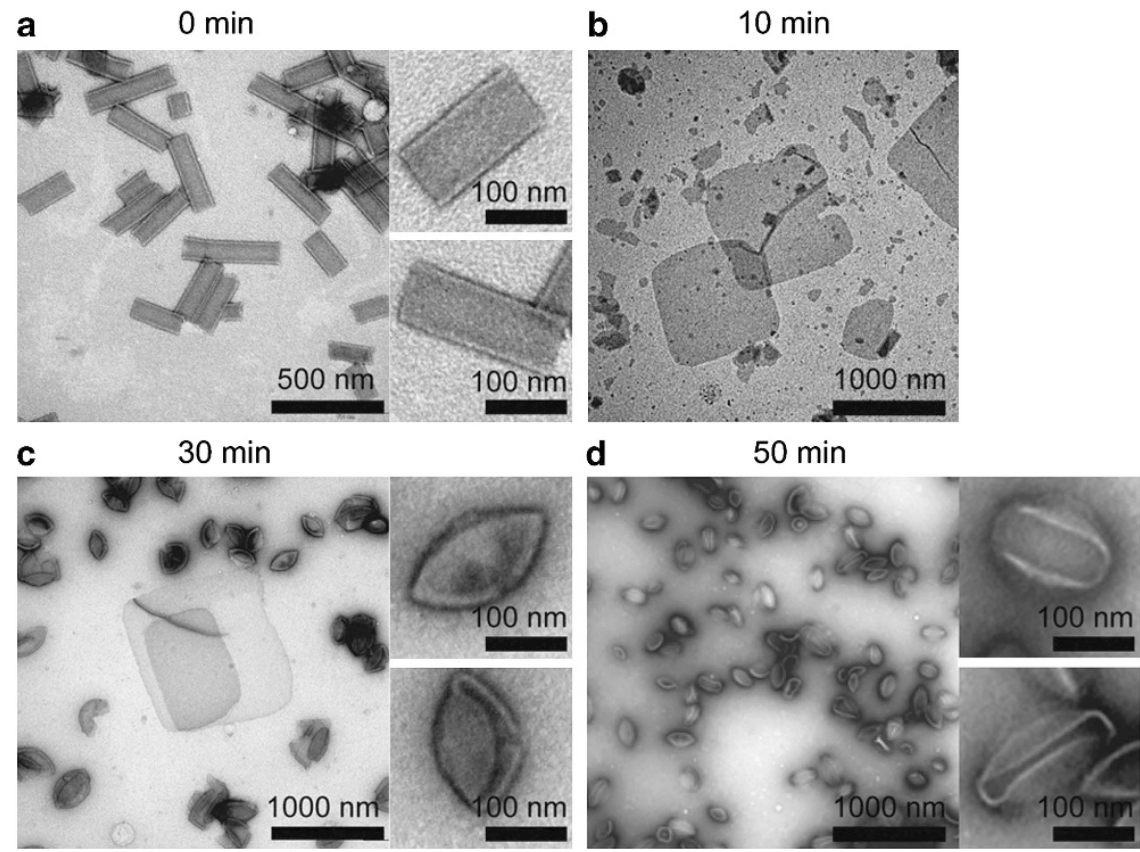

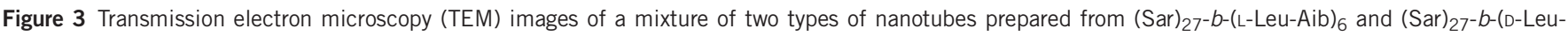
Aib) 6 upon heating at $90^{\circ} \mathrm{C}$ for the specified times. The dispersions were negatively stained using $2 \%$ uranyl acetate (reprinted with permission from Figure 1 in Ueda et al. ${ }^{28}$ Copyright 2011 The Royal Society of Chemistry).

Therefore, morphologic changes can be induced through small changes in the hydrophobic-hydrophilic balance of the polymer.

\section{Photosensitive self-assembly}

A photosensitive assembly was also prepared using the photosensitivity characteristics of azobenzene. The structure of an azobenzenelinked symmetrical gemini helical peptide was reversibly transformed between $\mathrm{Z}$ and $\mathrm{U}$ structures through ultraviolet and visible light irradiation, and the morphology of the resulting molecular assembly changed between nanofibers and nanospheres in solution at $\mathrm{pH} 3.0$ and between nanospheres and vesicles in solution at $\mathrm{pH} 8.0 .^{23}$

\section{Thermosensitive assembly}

Based on thermosensitive geleation of amphiphilic poly(ethylene glycol) (PEG)- $b$-poly(alkyl-L-glutamate), ${ }^{24,25}$ PEG- $b$-poly-L-EG 2 Glu with a thermosensitive $\operatorname{poly}(\gamma$-(2-methoxyethoxy)esteryl-L-glutamate) (poly-L-EG $\mathrm{E}_{2} \mathrm{Glu}$ ) block was synthesized, and its self-assembly was evaluated. ${ }^{26}$ With higher temperatures in solution, the poly-L-EG ${ }_{2} \mathrm{Glu}$ unit released its hydrated water, and a worm-like micelle was formed. Under heat, the poly-L-EG $\mathrm{E}_{2} \mathrm{Glu}$ unit changed its secondary structure from a helical conformation to a $\beta$-sheet structure, which preferred a parallel or antiparallel copolymer arrangement, and the conformation was transitioned to nanoribbons.

The repeated peptide sequence of L-leucine (Leu) and 2-aminoisobutylic acid (Aib) forms a helical structure. ${ }^{15}$ Amphiphilic poly(Sar)-b-(Leu-Aib) $)_{x}$ was self-assembled, and the micelle and vesicle assemblies were prepared by changing the hydrophobic-hydrophilic balance of the amphiphile. ${ }^{27}$ (Sar) ${ }_{27}-b$-(Leu-Aib) 6 yielded a curved sheet structure when the polymer film coated on the test tube was sonicated at room temperature in an aqueous solution (Figure 2a). The curved sheet shrank upon heating the solution to $90^{\circ} \mathrm{C}$ for $10 \mathrm{~min}$ and transformed into a tubular structure (Figure $2 \mathrm{~b}$ ) because the regularly aligned helical hydrophobic unit was exposed on the edge of the curved sheet structure; therefore, the sheet is thermodynamically metastable. The sheet was irreversibly transformed into a thermodynamically stable tubular structure through heating. The uniform-size tubular structure that supports the morphology is regulated by a helical hydrophobic block, which is regularly aligned in the assembly. Importantly, a metastable hydrophobic surface remained at the open mouth of the tubular structure. The exposed 


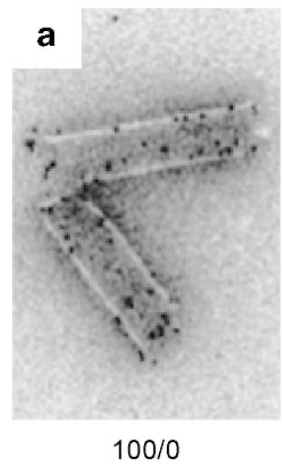

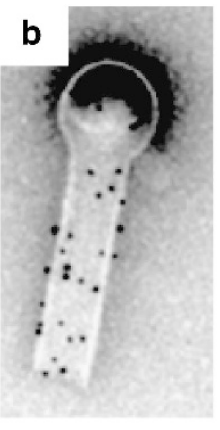

$80 / 20$

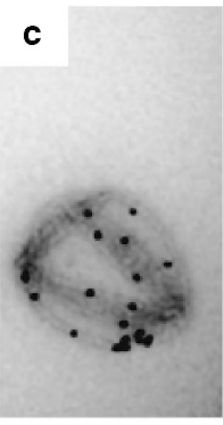

$50 / 50$

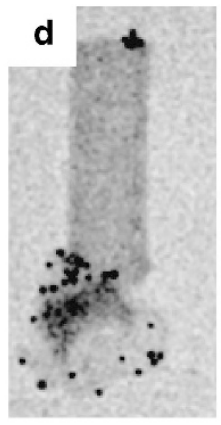

$20 / 80$

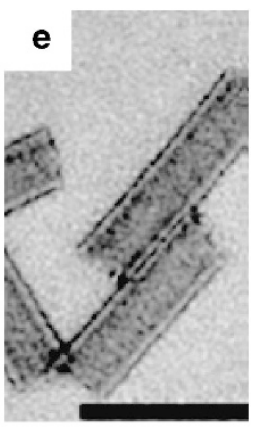

$0 / 100$

sSLL + SLL / SDL $(w / w)$

(the content of $\mathbf{S S L L}$ is $10 \%$ of $\mathbf{S S L L}+\mathbf{S L L}$ )

Figure 4 Distribution of gold nanoparticles $(10 \mathrm{~nm})$ on the surface of the molecular assemblies prepared from mixtures of various $\mathbf{s S L L}+\mathrm{SLL} \mathbf{S D L}\left(\mathrm{ww} \mathrm{w}^{-1}\right.$ ) ratios: (a) 100/0, (b) 80/20, (c) 50/50, (d) 20/80 and (e) 0/100 (the sSLL content is $10 \%$ of SLL + sSLL). The polymer dispersions were negatively stained by $2 \%$ uranyl acetate. Scale bar: $200 \mathrm{~nm}$ (reprinted with permission from Figure 4 in Ueda et al. ${ }^{29}$ Copyright 2011 The Royal Society of Chemistry).
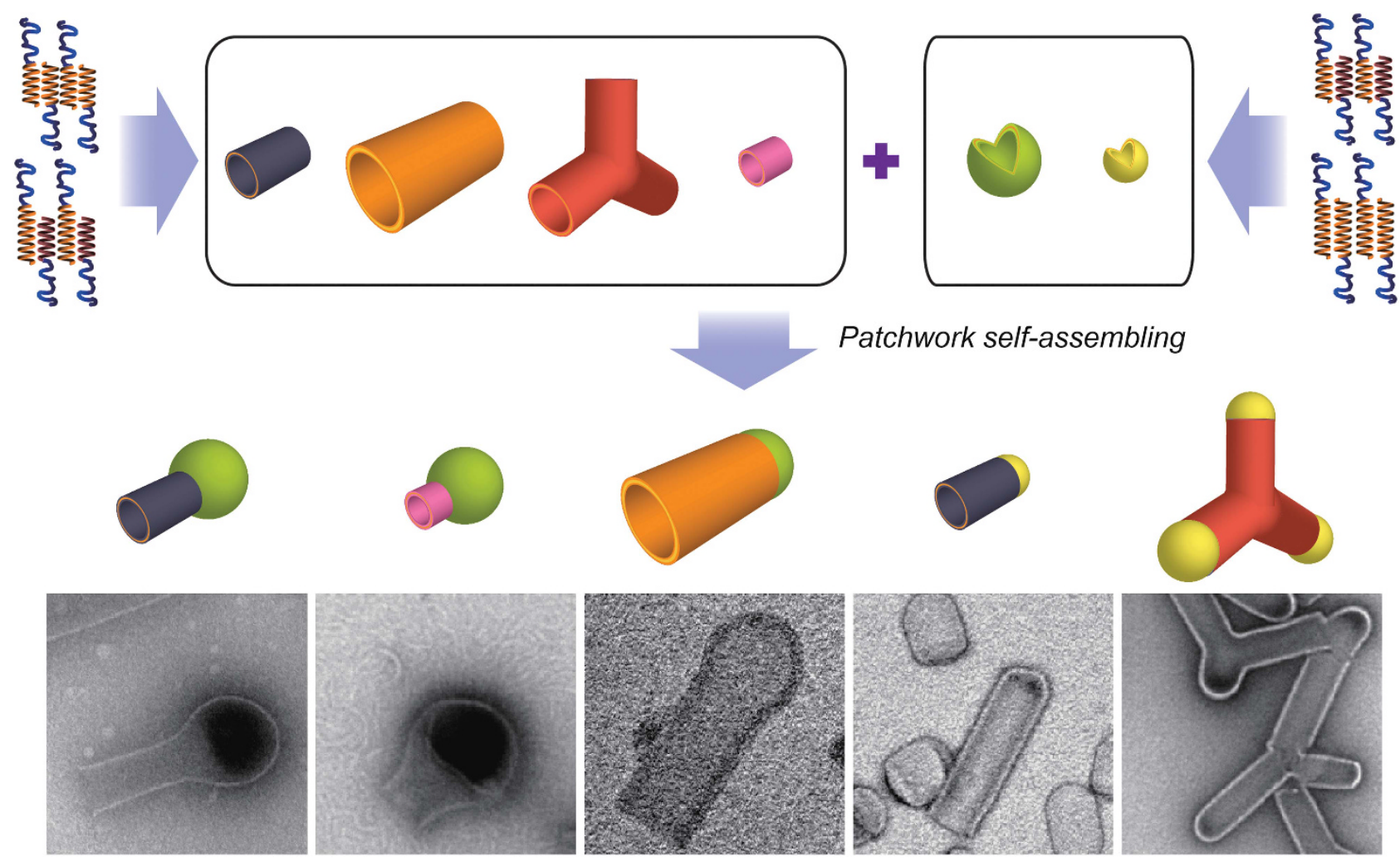

Patchwork self-assembling
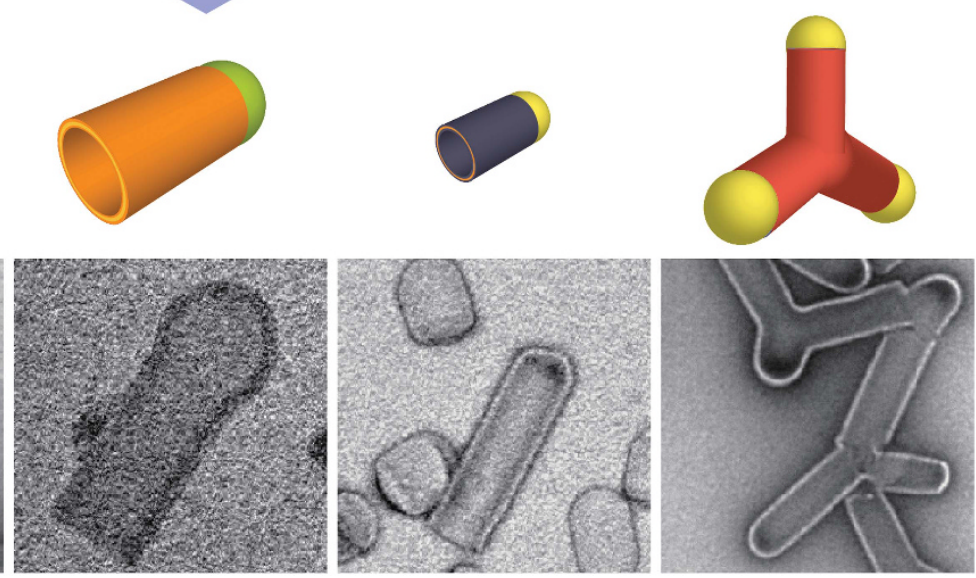

Figure 5 Transmission electron microscopy (TEM) images of the molecular assemblies prepared via patchwork self-assembly techniques.

hydrophobic region can be used as a glue to connect the tubes, and a prolonged tubular assembly was prepared by gradually heating the solution. Furthermore, a three-way nanotube was also prepared from the $7: 3\left(\mathrm{~mol} \mathrm{~mol}^{-1}\right)$ polypeptide mixture of $(\mathrm{Sar})_{27}-b$-(Leu-Aib $)_{6}$ and $(\text { Sar })_{10}-b$-(Leu-Aib) 6 (Figure 2c). ${ }^{27}$

\section{Using the left- and right-handed helical components}

Helical peptides can be designed to take either helicity through using $\mathrm{L}$ - or D-peptide residues in the sequence. For example, the repeated peptide sequence (L-Lue-Aib) and (D-Leu-Aib) forms a right- and left-handed helical conformation, respectively. Regardless of the rotational direction, the molecular assembly morphology did not change, and both $(\text { Sar })_{27}-b$-(L-Leu-Aib) ${ }_{6}$ and $(\text { Sar })_{27}-b$-(D-Leu-Aib) amphiphiles self-assembled and produced nanotubes. ${ }^{28}$ The tubular assemblies were stable at room temperature and $90^{\circ} \mathrm{C}$. However, the nanotube structure transformed into a vesicle when the two types of dispersions were mixed at an equimolar ratio, and the mixture was heated to $90{ }^{\circ} \mathrm{C}$ for $50 \mathrm{~min}$ (Figure 3). ${ }^{28}$ Detailed observations on the vesicle formation process upon heating to $50^{\circ} \mathrm{C}$ revealed that two types of peptide nanotubes were connected at a metastable open edge. The right- and left-handed helical peptides diffused throughout the assembly to cause membrane fusion because the assembly exhibits membrane fluidity, and the stereocomplex is thermodynamically stable. The mixing proceeded with time, and the tubular structure 
finally broke up into a planar square sheet. Upon heating to $90{ }^{\circ} \mathrm{C}$, the sheet transformed into a vesicle to shield the metastable hydrophobic edges of the sheet. Because the helical polypeptide mixture alignment differs from the unihelical polypeptide, the sheet is considered transformed into a vesicle.

Fusion of the tubular assembly was evaluated by changing mixing ratio of the $\mathrm{HOCH}_{2} \mathrm{CO}-(\mathrm{Sar})_{25}-b$-(L-Leu-Aib) ${ }_{6}-\mathrm{OMe}$ (SLL) and $\mathrm{HOCH}_{2} \mathrm{CO}-(\mathrm{Sar})_{25}-b$-(D-Leu-Aib) ${ }_{6}-\mathrm{OMe} \quad$ (SDL) dispersions. ${ }^{29}$ Depending on the mixing ratio of the amphiphile, nanotubes, vesicles and round-bottomed flask-shaped assemblies formed. Next, lipoic acid was introduced to the sarcosine N-terminal end of the $(\text { Sar })_{25}-b$-(L-Leu-Aib) 6 polypeptide, and a molecular assembly was prepared from mixing the liponic acid-modified (L-Leu-Aib) ${ }_{6}-b$ $(\text { Sar })_{25}(\mathbf{s S L L})+$ SLL and SDL at an arbitrary ratio (the sSLL composed $10 \%$ of SLL + sSLL). ${ }^{29}$ After the molecular assembly dispersion was dropped on a $\mathrm{Cu}$ grid for TEM measurements, an aliquot of gold nanoparticles $10 \mathrm{~nm}$ in diameter was added. From the TEM images, the polymer constituents in the assemblies were determined by evaluating the location of the $\mathrm{Au}$ nanoparticle bound to liponic acid. On the nanotube surface from sSLL + SLL/ $\mathrm{SDL}=100 / 0$ and $0 / 100\left(\mathrm{ww}^{-1}\right)$, adhered and unadhered gold nanoparticles were observed in a tubular assembly, respectively (Figures $4 \mathrm{a}$ and e). Vesicle surfaces prepared from a 50/50 $\left(\mathrm{ww}^{-1}\right)$ polypeptide mixture were uniformly coated by gold nanoparticles, which indicates that both polypeptides were well mixed in the assembly (Figure 4c). Importantly, for the round-bottomed flask prepared from the $80 / 20\left(\mathrm{w} \mathrm{w}^{-1}\right)$ polypeptides mixture, gold nanoparticles were distributed throughout the entire assembly surface (Figure 4b). On the other hand, the gold nanoparticles were localized to the spherical portion when the round-bottomed flask-shaped assembly was prepared using a 20/80 $\left(\mathrm{ww}^{-1}\right)$ mixture (Figure $4 \mathrm{~d}$ ). These results indicate that the spherical portion is constructed by a stereocomplex of the right- and left-handed helical polypeptides through an equimolar mixture, and the tubular portion in the phase-separation condition occurs owing to excess polypeptide chain.

Preparing molecular assemblies with various morphologies

Molecular assemblies with a more complex structure were prepared from amphiphilic polypeptides with a helical hydrophobic component using the combined methods described above. For example, dumbbell-, maracas- and capsule-shaped assemblies were prepared by sealing both open ends of the nanotube with different diameter vesicles (Figure 5). ${ }^{30}$ This unique self-assembly process is driven by stereocomplex formation of the helical amphiphiles and is referred to as 'patchwork self-assembly'.

Preparing micelles with a controlled diameter

Poly(L-lactic acid) (PLLA) is a representative biodegradable polymer and forms a helical structure. ${ }^{31}$ A molecular assembly prepared from amphiphilic polydepsipeptides of PLLA- $b$-poly(Sar) is referred to as a 'lactosome'. Where a helical PLLA block was regularly aligned in an assembly, micelle-, vesicle- and lamella-shaped assemblies were prepared by controlling the hydrophilic-hydrophobic balance of the polymer. $^{32}$

A linear amphiphilic polydepsipeptide of (Sar) $60-90-b$-(L-lactic acid $)_{30}$ forms a polymeric micelle with the diameter ca. $34 \mathrm{~nm}$. Branched-type amphiphilic polymers with two or three hydrophilic poly $(\mathrm{Sar})$ chains and one hydrophobic block $\left(\mathrm{A}_{2} \mathrm{~B}:\left((\mathrm{Sar})_{26}\right)_{2}-b-\right.$ $(\mathrm{LA})_{33} ; \mathrm{A}_{3} \mathrm{~B}$ : $\left.(\mathrm{Sar})_{23}\right)_{3}-b-(\mathrm{LA})_{30}-b-(\mathrm{LA})_{30} ; \mathrm{A}$ : hydrophilic block; B: hydrophobic block) were newly synthesized, and their self-assembly was evaluated to examine the relationship between polymer architecture and resulting assembly morphology. ${ }^{33}$ In an aqueous solution, spherical and worm-like micelles were generated from $\mathrm{A}_{3} \mathrm{~B}$ and $A_{2} B$-type polymers, respectively. Depending on the increased surface density of the poly(Sar) chains, the micelle diameter from the $\mathrm{A}_{3} \mathrm{~B}$-type polymer decreased to ca. $20 \mathrm{~nm}$. On the other hand, the $\mathrm{A}_{2} \mathrm{~B}$-type polymer is branched three ways at the nitrogen atom and forms a fan-like geometry. To shield the hydrophobic molecular surface, the planar-like $\mathrm{A}_{2} \mathrm{~B}$ polymer is considered favorably stacked in the worm-like morphology.

The hydrophobic PLLA unit in $\mathrm{AB}$ - and $\mathrm{A}_{3} \mathrm{~B}$-type polymers was a 30 mer. A molecular assembly was prepared from a mixture of the $\mathrm{AB}$ - and $\mathrm{A}_{3} \mathrm{~B}$-type polymers with an arbitrary ratio and was evaluated through dynamic light scattering and transmission electron microscopy measurements. As a result, polymeric micelles with sizes ranging from $35 \mathrm{~nm}\left(\mathrm{AB} / \mathrm{A}_{3} \mathrm{~B}=100 / 0\right)$ to $20 \mathrm{~nm}\left(\mathrm{AB} / \mathrm{A}_{3} \mathrm{~B}=0 / 100\right)$ were prepared, and the micelle size was linearly correlated with the mixing ratio (Figure 6). Through regulated alignment of the helical hydrophobic block in the micelle, the size of the polymeric micelle can be controlled with nanometer precision based on the micelle surface density.

Amphiphilic polymers with a helical hydrophobic block are unique, which facilitates preparation of molecular assemblies that have not been prepared using conventional approaches.

\section{APPLYING HELICAL POLYMER-BASED MOLECULAR ASSEMBLIES TO TUMOR-TARGETED IN VIVO IMAGING AND DRUG DELIVERY SYSTEMS}

Using nano-ordered assemblies for tumor-targeted drug delivery At tumor regions, blood vessels exhibit holes due to rapid cell proliferation, forming a leaky environment. Coupled with an undeveloped lymph system, nanoparticles passively accumulate and are retained at the tumor. This phenomenon is referred to as the

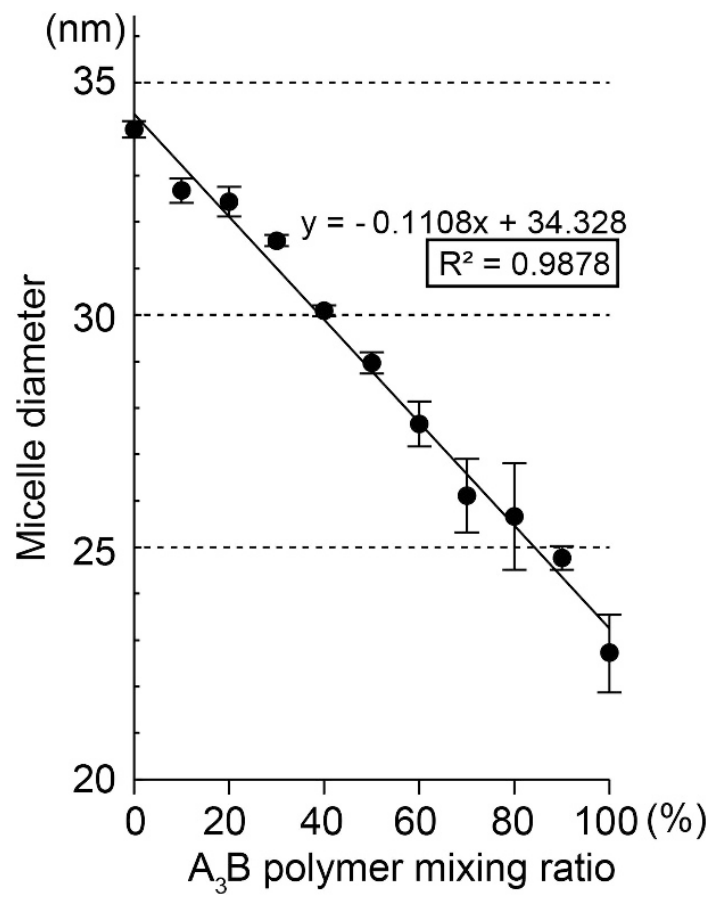

Figure 6 Relationship between the diameter of the polymeric micelle and mixing ratio of $\mathrm{AB}$ - and $\mathrm{A}_{3} \mathrm{~B}$-type amphihilic polydepsipeptides. The micelle diameter shows a linear correlation $\left(R^{2}=0.99\right)$ with the polymer mixing ratio. 

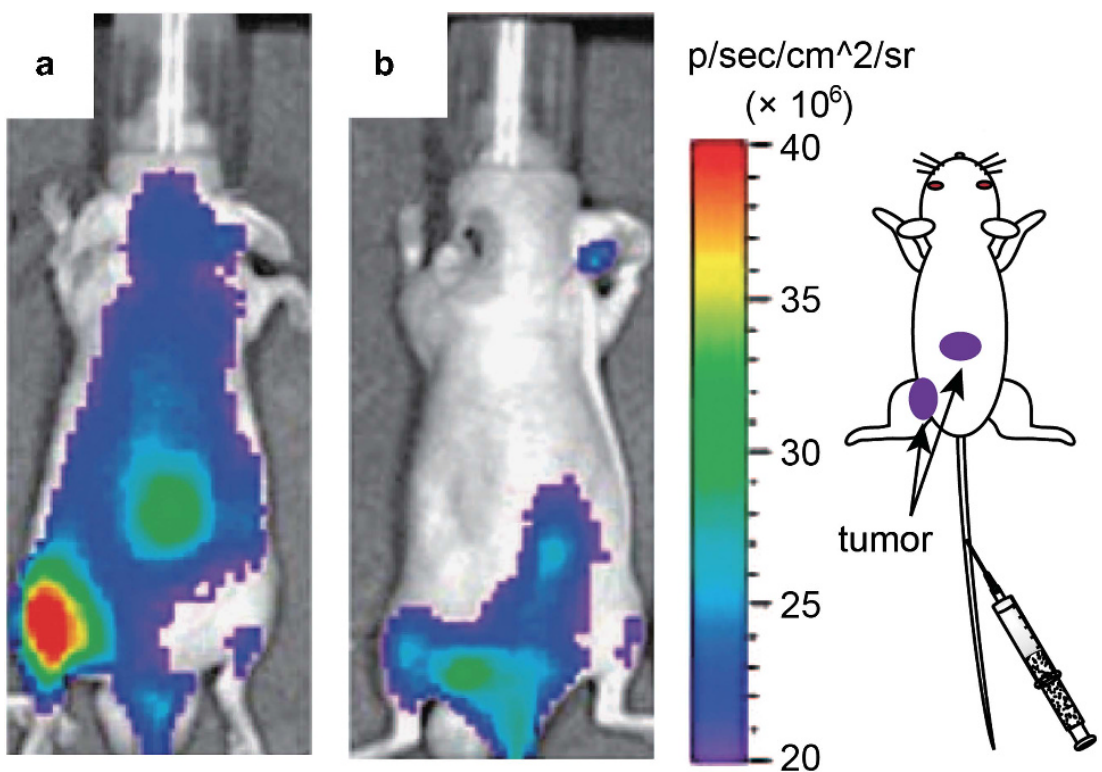

Figure 7 Images collected using a in vivo near-infrared fluorescence (NIRF) imaging system after $24 \mathrm{~h}$ from (a) an indocyanine green (ICG)-labeled peptide micelle and (b) an ICG-labeled vesicle dose from the mice tail vein. SUIT-2/pEF/luc cells were transplanted at the right femoral region of the mice. (NIRF images are reprinted with permission from Figures 7 and 8 in Tanisaka et al..$^{50}$ Copyright 2008 American Chemical Society).

a

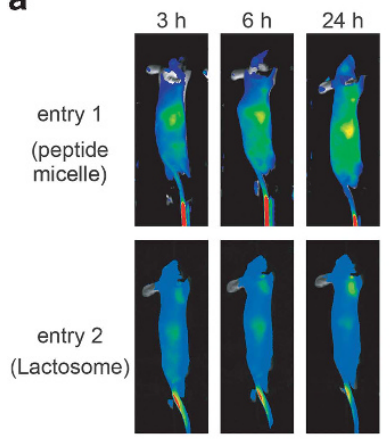

b

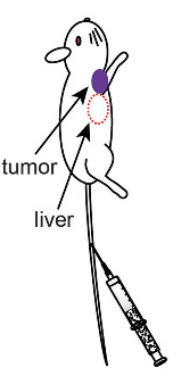

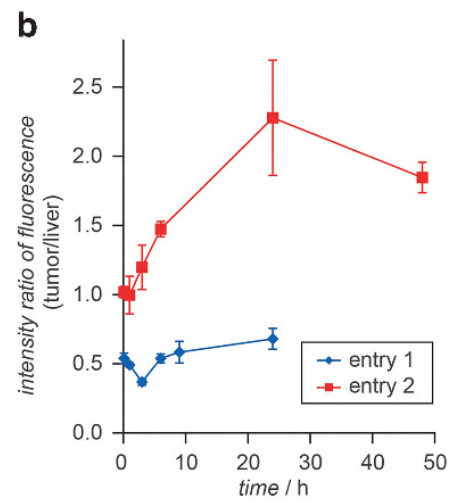

Figure 8 (a) Near-infrared fluorescence (NIRF) images at 3, 6 and $24 \mathrm{~h}$ from the indocyanine green (ICG)-labeled lactosome dose. The SUIT-2/pEF/luc cells were transplanted at the right shoulder region of the mice. (b) Time courses of the fluorescein signal intensity ratio at the tumor against the liver (reprinted from Figure 2 in Makino et al. ${ }^{51}$ with permission from Elsevier. Copyright 2009 Elsevier Ltd).

enhanced permeability and retention (EPR) effect $^{34}$ and is one of the most frequently used approaches in nanoparticles DDSs. ${ }^{35,36}$

Nanoparticles prepared from amphiphilic polypeptides or polydepsipeptides are likely biocompatible and, thus, effective carriers for tumor-targeted DDSs. Various types of antitumor drug-encapsulated micelles have been prepared, ${ }^{37-39}$ and certain PEGylated peptidebased assemblies are under evaluation in clinical trials. ${ }^{40}$ Recently, functional DDSs developed using stimulus-responsive polypeptidebased assemblies were actively examined. ${ }^{41-43}$ Nanoparticles can also be applied as imaging probe carriers. Typically, biodistribution of the nanoparticles remains unchanged even if different compounds are encapsulated. Therefore, the heterogeneous and localized response to nanoparticle-based therapy can be identified and visualized using imaging techniques. ${ }^{44} \mathrm{~A}$ 'theranostic' nanoparticle is a portmanteau of therapeutics and diagnostics and is considered a key concept for personalized medicine in the future. ${ }^{45,46}$
Molecular assemblies prepared from poly(Sar)-b-helical polypeptide amphiphiles

Sarcosine ( $N$-methyl glycine) is natural amino acid, and its polymer, which can be easily synthesized using the sarcosine $\mathrm{N}$-carboxy anhydride polymerization method, is highly soluble in aqueous solutions. ${ }^{47-49}$ Therefore, the authors focused especially on poly(Sar) as a hydrophilic component of amphiphilic polypeptides and polydepsipeptides to form molecular assemblies for medicinal use.

For example, for the poly(sarcosine) $\mathrm{N}$-terminal end of (sarcosine $)_{63}-b$ - $(\gamma \text {-methyl-L-glutamate })_{20}$ (PSar63-PMLG20), indocyanine green (ICG) was modified through a transesterification reaction, and an ICG-labeled peptide micelle and vesicle with the diameters ca. 40 and $100 \mathrm{~nm}$ were prepared from the mixture of PSar93-PMLG12 and ICG-PSar63-PMLG20. ${ }^{50}$ Figure 7 shows in vivo images collected $24 \mathrm{~h}$ after administration. Low accumulation in the liver indicates that 
the PSar chain has an important role in suppressing undesired nanoparticle accumulation by the reticuloendothelial system, and both near-infrared fluorescence-labeled probes accumulated at a transplanted tumor region due to the EPR effect. When ICG-labeled peptide micelle biodistribution was compared with vesicles, the ICG-labeled micelle showed prolonged blood circulation behavior with high ICG signal intensity detected at the tumor region. These results indicate that the nanoparticle size is crucial for reticuloendothelial system detection and the EPR effect. Furthermore, Dy776labeled peptide micelles with a ca. $32 \mathrm{~nm}$ diameter were also prepared from (L-Leu-Aib) $)_{8}-b-(\text { Sar })_{60}$ by mixing $1.5 \mathrm{~mol} \%$ of Dy776-labeled (L-Leu-Aib) 8 . The micelle accumulated at the transplanted tumor region. ${ }^{51}$ The peptide micelle with an outer surface covered by poly(Sar) was successfully delivered to the transplanted tumor region.

Molecular assemblies prepared from amphiphilic polypeptides with helical hydrophobic and hydrophilic poly(Sar) blocks can also be used as DDS carriers. ${ }^{16,52} \mathrm{Poly}(\mathrm{Sar})$ is likely a hydrophilic polymer alternative to PEG and can be used for medicinal applications.

\section{'Lactosome' polymeric micelles prepared from amphiphilic} polydepsipeptides with excellent blood circulation behavior Polymeric micelles with a ca. $35 \mathrm{~nm}$ diameter were prepared from (L-lactic acid) ${ }_{30}-b$-(Sar $)_{70}$ and are referred to as 'lactosomes.' ${ }^{32}$ ICG-labeled (L-lactic acid) 30 was synthesized through chemical modification of the PLLA terminal end using an indocyanine green derivative of ICG-sulfo-OSu. The ICG-labeled lactosome was prepared from a 98.5:1.5 $\left(\mathrm{mol} \mathrm{mol}^{-1}\right)$ mixture of (Sar) $)_{90}-b$-(L-lactic acid $)_{30} \quad\left(\mathrm{PS}_{90} \text {-block-PLLA } 30 \text { and ICG-labeled (L-lactic acid) }\right)_{30}$ (ICG-PLLA); it was then injected into the tumor-transplanted model mice at the tail vein. Similar to the ICG-labeled peptide micelle (diameter: ca. $32 \mathrm{~nm}$ ) generated from $(\text { Sar })_{60}-b$-(L-Leu-Aib) 8 (Figure 8, entry 1), a high fluorescein signal was observed at the tumor region $24 \mathrm{~h}$ after administration (entry 2). Importantly, the signal intensity detected in the liver was extremely low, ${ }^{51}$ and HepG2 from the human hepatocellular liver carcinoma cell line, which was orthotopically transplanted at the liver, was clearly detected by the ICG-labeled lactosome. ${ }^{51}$ The lactosome and peptide micelle diameters did not differ significantly, and their outer surfaces were covered with poly(Sar) chains. Their main structural difference is the chemical structure of the hydrophobic block. (L-Leu-Aib) ${ }_{8}$ and PLLA chains form $\alpha$ - and $30_{10}$-helical structures, and their diameters are 1.4 and $0.62 \mathrm{~nm}$, respectively. ${ }^{51}$ Therefore, the surface density of the poly(Sar) chains in the lactosome were simply calculated at five times higher than the peptide micelle. Lactosome recognition by reticuloendothelial system is considered suppressed by the densely aligned poly(Sar) brush on the surface. Lactosomes are considered nano-ordered carrier candidates for drug and/or imaging agent delivery.

Lactosomes can also be used as nano-ordered carriers for internal radiation therapy drugs. ${ }^{53}$ Radioisotopes that emit $\beta^{-}$-rays, such as ${ }^{131} \mathrm{I}$ and ${ }^{90} \mathrm{Y}$, are typically used for radiation therapy; therefore, ${ }^{131}$ I-labeled lactosomes ( ${ }^{131} \mathrm{I}$-lactosome) with a ca. $35 \mathrm{~nm}$ diameter were prepared by mixing a hydrophobic ${ }^{131}$ I-labeled L-lactic acid 30 mer $\left({ }^{131} \text { I-BzPLLA) and poly(sarcosine }\right)_{64}$-block-poly(L-lactic acid $)_{30}\left(\operatorname{poly}(\text { Sar })_{64}\right.$-block-PLLA 30$) .{ }^{53}{ }^{131}$ I-lactosome exhibited a high cytoreductive effect in combined percutaneous ethanol injection therapy ${ }^{54,55}$ likely because ${ }^{131}$ I-lactosome effectively accumulates at the surviving tumor region, where inflammation and neovasculation are induced through the percutaneous ethanol injection therapy pretreatment.
For the development of lactosomes as nano-ordered carriers for DDS, controlling the rate of drug release from lactosomes is another important consideration. During ${ }^{131} \mathrm{I}$-lactosome preparation, ${ }^{131} \mathrm{I}$ is hydrophobized by attaching the PLLA of the 30 mer for stabilized encapsulation into the lactosome. Next, ICG-sulfo-OSu reacted with three types of 28-31 mer poly(lactic acid)s, and ICG-PLLA, ICG-poly(D-lactic acid) and ICG-poly(DL-lactic acid) were synthesized. ICG-lactosomes were prepared by mixing (Sar) $)_{70}-b$-(L-lactic acid $)_{30}$ with these three ICG-labeled poly(lactic acid)s. ${ }^{56}$ Poly(D-lactic acid) takes a right-handed helical structure, and therefore, a stereocomplex is formed between the ICG-poly(D-lactic acid) and PLLA unit of the amphiphile. The stereocomplex stabilizes the micelle, and the rate of ICG-poly(D-lactic acid) release from the lactosome became slower than the ICG-PLLA. On the other hand, poly(DL-lactic acid) does not take a specific high-ordered structure. ICG-poly(DL-lactic acid) could not form stable hydrophobichydrophobic interactions with the amphiphile and was released from lactosome in a relatively rapid manner. ${ }^{56}$

The methodology for controlling the speed of encapsulated drug release from a nano-ordered carrier is not a simple problem. However, using stability of interhelical interactions is considered a unique approach to controlling the release rate of the encapsulated compounds.

\section{CONCLUSION}

The author prepared molecular assemblies with specific morphologies and functions using helical hydrophobic polymers as a component of the amphiphile. These unique characteristics are derived from the regularly aligned hydrophobic polymer in the assembly at the molecular level. Recently, designing materials at the molecular level has been considered important for functionalization. Therefore, 'patch-work self-assembly' is expected to become a powerful tool for designing new materials.

In this study, several types of polypeptide and polydepsipeptides sequences were used as helical hydrophobic blocks of amphiphilic polymers. Molecular assemblies with more complex structures and specific functions are expected to be prepared by selecting the appropriate helical component as the hydrophobic unit of the amphiphile.

\section{ACKNOWLEDGEMENTS}

The research in this paper was mainly performed at the Department of Material Chemistry, Graduate School of Engineering, Kyoto University, Japan. I am grateful to and thank Prof Shunsaku Kimura and all his collaborators. This study is a part of a joint research project focused on the development of basic technology for establishing a COE for nanomedicine through the Kyoto City Collaboration of Regional Entities for Advancing Technology Excellence (CREATE), Japan Science and Technology Agency, is part of the project entitled, 'Development of PET Probe for Imaging Solid Tumors Using Nanocarriers' under the contract of Innovation plaza Kyoto, Japan Science and Technology Agency, and is part of the Innovative Techno-Hub for Integrated Medical Bio-Imaging of the Project for Developing Innovation Systems from the Ministry of Education, Culture, Sports, Science and Technology (MEXT).

1 Davis, M. E., Chen, Z. \& Shin, D. M. Nanoparticle therapeutics: an emerging treatment modality for cancer. Nat. Rev. Drug Discov. 7, 771-782 (2008).

2 Hao, R., Xing, R. J., Xu, Z. C., Hou, Y. L., Gao, S. \& Sun, S. H. Synthesis, functionalization, and biomedical applications of multifunctional magnetic nanoparticles. Adv. Mater. 22, 2729-2742 (2010).

3 Horcajada, P., Gref, R., Baati, T., Allan, P. K., Maurin, G., Couvreur, P., Ferey, G., Morris, R. E. \& Serre, C. Metal-organic frameworks in biomedicine. Chem. Rev. 112 1232-1268 (2012). 
4 Kumari, A., Yadav, S. K. \& Yadav, S. C. Biodegradable polymeric nanoparticles based drug delivery systems. Colloid Surf. B 75, 1-18 (2010)

5 Li, Z. X., Barnes, J. C., Bosoy, A., Stoddart, J. F. \& Zink, J. I. Mesoporous silica nanoparticles in biomedical applications. Chem. Soc. Rev. 41, 2590-2605 (2012).

6 Gabizon, A., Catane, R., Uziely, B., Kaufman, B., Safra, T., Cohen, R., Martin, F. Huang, A. \& Barenholz, Y. Prolonged circulation time and enhanced accumulation in malignant exudates of doxorubicin encapsulated in polyethylene-glycol coated liposomes. Cancer Res. 54, 987-992 (1994).

7 Gabizon, A., Shmeeda, H. \& Barenholz, Y. Pharmacokinetics of pegylated liposomal doxorubicin-review of animal and human studies. Clin. Pharmacokinet. 42, 419-436 (2003)

8 Nagarajan, R. Molecular packing parameter and surfactant self-assembly: the neglected role of the surfactant tail. Langmuir 18, 31-38 (2002).

9 Cabral, H., Matsumoto, Y., Mizuno, K., Chen, Q., Murakami, M., Kimura, M., Terada, Y., Kano, M. R., Miyazono, K., Uesaka, M., Nishiyama, N. \& Kataoka, K. Accumulation of sub-100 nm polymeric micelles in poorly permeable tumours depends on size. Nat. Nanotechnol. 6, 815-823 (2011).

10 Tang, L., Gabrielson, N. P., Uckun, F. M., Fan, T. M. \& Cheng, J. J. Size-dependent tumor penetration and in vivo efficacy of monodisperse drug-silica nanoconjugates. Mol. Pharm. 10, 883-892 (2013).

11 Discher, B. M., Won, Y. Y., Ege, D. S., Lee, J. C. M., Bates, F. S., Discher, D. E. \& Hammer, D. A. Polymersomes: tough vesicles made from diblock copolymers. Science 284, 1143-1146 (1999).

12 Letchford, K. \& Burt, H. A review of the formation and classification of amphiphilic block copolymer nanoparticulate structures: micelles, nanospheres, nanocapsules and polymersomes. Eur. J. Pharm. Biopharm. 65, 259-269 (2007).

13 Tada, Y., Morita, T., Umemura, J., Iwamoto, M. \& Kimura, S. Photoresponsive change of the surface potential generated by helical peptide self-assembled monolayers. Polym. J. 37, 599-607 (2005)

14 Yanagisawa, K., Morita, T. \& Kimura, S. Efficient photocurrent generation by selfassembled monolayers composed of 3(10)-helical peptides carrying linearly spaced naphthyl groups at the side chains. J. Am. Chem. Soc. 126, 12780-12781 (2004).

15 Takeda, K., Morita, T. \& Kimura, S. Effects of monolayer structures on long-range electron transfer in helical peptide monolayer. J. Phys. Chem. B 112, 12840-12850 (2008)

16 Kidchob, T., Kimura, S. \& Imanishi, Y. Amphiphilic poly(Ala)-b-poly(Sar) microspheres loaded with hydrophobic drug. J. Control Rel. 51, 241-248 (1998).

17 Kimura, S., Kidchob, T. \& Imanishi, Y. Controlled release from amphiphilic polymer aggregates. Polym. Adv. Technol. 12, 85-95 (2001).

18 Fujita, K., Kimura, S., Imanishi, Y., Rump, E. \& Ringsdorf, H. Supramolecula assembly using helical peptides. Adv. Biophys. 34, 127-137 (1997).

19 Rodriguez-Hernandez, J. \& Lecommandoux, S. Reversible inside-out micellization of $\mathrm{pH}$-responsive and water-soluble vesicles based on polypeptide diblock copolymers. J. Am. Chem. Soc. 127, 2026-2027 (2005).

20 Chen, L. L., Chen, T., Fang, W. X., Wen, Y., Lin, S. L., Lin, J. P. \& Cai, C. H. Synthesis and $\mathrm{pH}$-Responsive '"chizophrenic' aggregation of a linear-dendron-like polyampholyte based on oppositely charged polypeptides. Biomacromolecules 14, 4320-4330 (2013)

21 Uesaka, A., Ueda, M., Makino, A., Imai, T., Sugiyama, J. \& Kimura, S. Self-assemblies of triskelion $\mathrm{A}(2) \mathrm{B}$-type amphiphilic polypeptide showing $\mathrm{pH}$-responsive morphology transformation. Langmuir 28, 6006-6012 (2012).

22 Uesaka, A., Ueda, M., Makino, A., Imai, T., Sugiyama, J. \& Kimura, S. Morphology control between twisted ribbon, helical ribbon, and nanotube self-assemblies with His-containing helical peptides in response to $\mathrm{pH}$ change. Langmuir 30, 1022-1028 (2014).

23 Chen, C. S., Xu, X. D., Li, S. Y., Zhuo, R. X. \& Zhang, X. Z. Photo-switched selfassembly of a gemini alpha-helical peptide into supramolecular architectures. Nanoscale 5, 6270-6274 (2013).

24 Chen, C. Y., Wang, Z. H. \& Li, Z. B. Thermoresponsive polypeptides from pegylated poly-L-glutamates. Biomacromolecules 12, 2859-2863 (2011).

25 Cheng, Y. L., He, C. L., Xiao, C. S., Ding, J. X., Zhuang, X. L., Huang, Y. B. \& Chen, X. $S$. Decisive role of hydrophobic side groups of polypeptides in thermosensitive gelation. Biomacromolecules 13, 2053-2059 (2012).

26 Shen, J. Y., Chen, C. Y., Fu, W. X., Shi, L. Q. \& Li, Z. B. Conformation-specific self assembly of thermo-responsive poly(ethylene glycol)-b-polypeptide diblock copolymer Langmuir 29, 6271-6278 (2013).

27 Kanzaki, T., Horikawa, Y., Makino, A., Sugiyama, J. \& Kimura, S. Nanotube and three way nanotube formation with nonionic amphiphilic block peptides. Macromol. Biosci. 8, 1026-1033 (2008).

28 Ueda, M., Makino, A., Imai, T., Sugiyama, J. \& Kimura, S. Transformation of peptide nanotubes into a vesicle via fusion driven by stereo-complex formation. Chem Commun. 47, 3204-3206 (2011).

29 Ueda, M., Makino, A., Imai, T., Sugiyama, J. \& Kimura, S. Tubulation on peptide vesicles by phase-separation of a binary mixture of amphiphilic right-handed and lefthanded helical peptides. Soft Matter 7, 4143-4146 (2011)

30 Ueda, M., Makino, A., Imai, T., Sugiyama, J. \& Kimura, S. Versatile peptide rafts for conjugate morphologies by self-assembling amphiphilic helical peptides. Polym. J. 45 509-515 (2013)

31 Fujiwara, T. \& Kimura, Y. Macromolecular organization of poly(L-lactide)-block polyoxyethylene into bio-inspired nano-architectures. Macromol. Biosci. 2, 11-23 (2002).
32 Makino, A., Yamahara, R., Ozeki, E. \& Kimura, S. Preparation of novel polyme assemblies, 'lactosome', composed of poly(L-lactic acid) and poly(sarcosine). Chem. Lett. 36, 1220-1221 (2007).

33 Makino, A., Hara, E., Hara, I., Ozeki, E. \& Kimura, S. Size control of core-shell-type polymeric micelle with a nanometer precision. Langmuir 30, 669-674 (2014).

34 Maeda, H., Wu, J., Sawa, T., Matsumura, Y. \& Hori, K. Tumor vascular permeability and the EPR effect in macromolecular therapeutics: a review. J. Control. Rel. 65, 271-284 (2000)

35 Maeda, H. Macromolecular therapeutics in cancer treatment: the EPR effect and beyond. J. Control Rel. 164, 138-144 (2012).

36 Maeda, H., Bharate, G. Y. \& Daruwalla, J. Polymeric drugs for efficient tumor-targeted drug delivery based on EPR effect. Eur. J. Pharm. Biopharm. 71, 409-419 (2009).

37 Harada, A. \& Kataoka, K. Novel polyion complex micelles entrapping enzyme molecules in the core: preparation of narrowly-distributed micelles from lysozyme and poly(ethylene glycol)-poly(aspartic acid) block copolymer in aqueous medium. Macromolecules 31, 288-294 (1998).

38 Kedar, U., Phutane, P., Shidhaye, S. \& Kadam, V. Advances in polymeric micelles for drug delivery and tumor targeting. Nanomed. Nanotechnol. Biol. Med 6, 714-729 (2010)

39 Lavasanifar, A., Samuel, J. \& Kwon, G. S. Poly(ethylene oxide)-block-poly(L-amino acid) micelles for drug delivery. Adv. Drug Deliv. Rev. 54, 169-190 (2002).

40 Gong, J., Chen, M. W., Zheng, Y., Wang, S. P. \& Wang, Y. T. Polymeric micelles drug delivery system in oncology. J. Control Rel. 159, 312-323 (2012).

41 Liang, J., Wu, W. L., Xu, X. D., Zhuo, R. X. \& Zhang, X. Z. pH responsive micelle selfassembled from a new amphiphilic peptide as anti-tumor drug carrier. Colloid Surf. $B$ 114, 398-403 (2014).

42 Lin, J. P., Zhu, J. Q., Chen, T., Lin, S. L., Cai, C. H., Zhang, L. S., Zhuang, Y. \& Wang, X. $S$. Drug releasing behavior of hybrid micelles containing polypeptide triblock copolymer. Biomaterials 30, 108-117 (2009).

43 Naik, S. S., Ray, J. G. \& Savin, D. A. Temperature- and pH-responsive self-assembly of Poly(propylene oxide)-b-poly(lysine) block copolymers in aqueous solution. Langmuir 27, 7231-7240 (2011)

44 Gustafson, T. P., Lim, Y. H., Flores, J. A., Heo, G. S., Zhang, F. W., Zhang, S. Y. Samarajeewa, S., Raymond, J. E. \& Wooley, K. L. Holistic assessment of covalently labeled core-shell polymeric nanoparticles with fluorescent contrast agents for theranostic applications. Langmuir 30, 631-641 (2014).

45 Park, J., Park, S., Kim, S., Lee, I. H., Saw, P. E., Lee, K., Kim, Y. C., Kim, Y. J. Farokhzad, O. C., Jeong, Y. Y. \& Jon, S. HER2-specific aptide conjugated magnetonanoclusters for potential breast cancer imaging and therapy. J. Mater. Chem. B 1 , 4576-4583 (2013)

46 Zou, P., Yu, Y. K., Wang, Y. A., Zhong, Y. Q., Welton, A., Galban, C., Wang, S. M. \& Sun, D. X. Superparamagnetic iron oxide nanotheranostics for targeted cancer cell imaging and pH-dependent intracellular drug release. Mol. Pharm. 7, 1974-1984 (2010).

47 Uejio, J. S., Schwartz, C. P., Duffin, A. M., England, A., Prendergast, D. \& Saykally, R J. Monopeptide versus monopeptoid: insights on structure and hydration of aqueous alanine and sarcosine via X-ray absorption spectroscopy. J. Phys. Chem. B 114 4702-4709 (2010)

48 Kricheldorf, H. R., von Lossow, C. \& Schwarz, G. Primary amine-initiated polymerizations of alanine-NCA and sarcosine-NCA. Macromol. Chem. Phys. 205, 918-924 (2004)

49 Sun, J. \& Zuckermann, R. N. Peptoid polymers: a highly designable bioinspired material. ACS Nano 7, 4715-4732 (2013).

50 Tanisaka, H., Kizaka-Kondoh, S., Makino, A., Tanaka, S., Hiraoka, M. \& Kimura, S. Near-infrared fluorescent labeled peptosome for application to cancer imaging. Bioconjugate Chem. 19, 109-117 (2008).

51 Makino, A., Kizaka-Kondoh, S., Yamahara, R., Hara, I., Kanzaki, T., Ozeki, E., Hiraoka, M. \& Kimura, S. Near-infrared fluorescence tumor imaging using nanocarrie composed of poly(L-lactic acid)-block-poly(sarcosine) amphiphilic polydepsipeptide. Biomaterials 30, 5156-5160 (2009).

52 Birke, A., Huesmann, D., Kelsch, A., Weilbacher, M., Xie, J., Bros, M., Bopp, T., Becker, C., Landfester, K. \& Barz, M. Polypeptoid-block-polypeptide copolymers: synthesis, characterization, and application of amphiphilic block copolypept(o)ides in drug formulations and miniemulsion techniques. Biomacromolecules 15, 548-557 (2014)

53 Hara, E., Makino, A., Kurihara, K., Ueda, M., Hara, I., Kawabe, T., Yamamoto, F. Ozeki, E., Togashi, K. \& Kimura, S. Radionuclide therapy using nanoparticle of I-131lactosome in combination with percutaneous ethanol injection therapy. J. Nanopart. Res. 15, 2131 (2013)

54 Ebara, M., Okabe, S., Kita, K., Sugiura, N., Fukuda, H., Yoshikawa, M., Kondo, F. \& Saisho, H. Percutaneous ethanol injection for small hepatocellular carcinoma: therapeutic efficacy based on 20-year observation. J. Hepatol. 43, 458-464 (2005) 55 Kawamura, R., Seki, T., Umehara, H., Ikeda, K., Inokuchi, R., Asayama, T., Yamaguchi, T., Takahashi, Y., Sakao, M., Lencioni, R. \& Okazaki, K. Combined treatment of large hepatocellular carcinoma with transcatheter arterial chemoembolization and percutaneous ethanol injection with a multipronged needle: experimental and clinica investigation. Cardiovasc. Interv. Radiol. 35, 325-333 (2012)

56 Makino, A., Hara, E., Hara, I., Yamahara, R., Kurihara, K., Ozeki, E., Yamamoto, F. \& Kimura, S. Control of in vivo blood clearance time of polymeric micelle by stereochemistry of amphiphilic polydepsipeptides. J. Control Rel. 161, 821-825 (2012) 


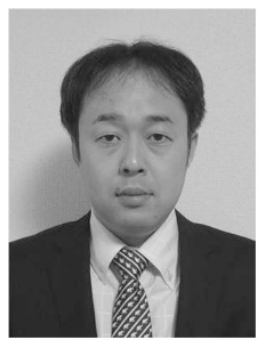

Akira Makino was born in Sapporo, Japan in 1977. He studied macromolecular chemistry in the research group of Prof Shiro Kobayashi at Kyoto University, where he received his BS degree in 2001 and MS in 2003. In 2006, he obtained his PhD degree of engineering from Kyoto University under the supervision of Prof Shiro Kobayashi and Prof Shunsaku Kimura, and started his postdoctoral research career at Shimadzu Corporation, Japan. He joined the Department of Material Chemistry, Graduate School of Engineering, Kyoto University (2007-2011), and then moved to the Department of Patho-Functional Bioanalysis, Graduate School of Pharmaceutical Sciences, Kyoto University (2011-2012). From 2012, he is an assistant professor of Biomedical Imaging Research Center, University of Fukui. His current research interests are synthesis of biocompatible functional materials based on the macromolecular chemistry. He was a recipient of Award for Encouragement of Research in Polymer Science, the Society of Polymer Science, Japan (2011). 